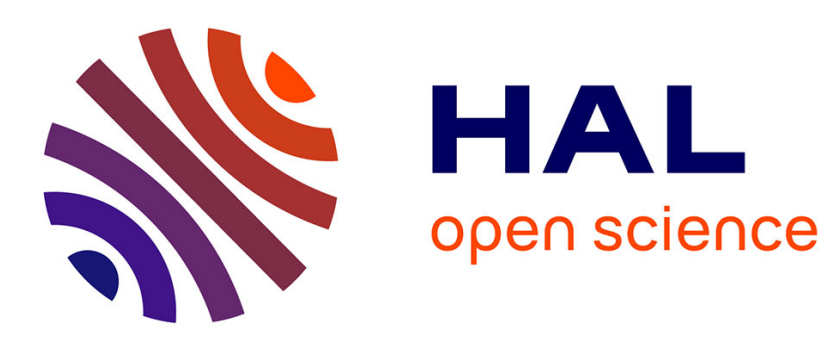

\title{
SOME NEW PERSPECTIVES IN BREMSSTRAHLUNG RESEARCH
}

A. Maquet, André Dubois, V. Véniard

\section{To cite this version:}

A. Maquet, André Dubois, V. Véniard. SOME NEW PERSPECTIVES IN BREMSSTRAHLUNG RESEARCH. Journal de Physique Colloques, 1987, 48 (C9), pp.C9-799-C9-809. 10.1051/jphyscol:19879141 . jpa-00227252

HAL Id: jpa-00227252

https://hal.science/jpa-00227252

Submitted on 1 Jan 1987

HAL is a multi-disciplinary open access archive for the deposit and dissemination of scientific research documents, whether they are published or not. The documents may come from teaching and research institutions in France or abroad, or from public or private research centers.
L'archive ouverte pluridisciplinaire HAL, est destinée au dépôt et à la diffusion de documents scientifiques de niveau recherche, publiés ou non, émanant des établissements d'enseignement et de recherche français ou étrangers, des laboratoires publics ou privés. 
SOME NEW PERSPECTIVES IN BREMSSTRAHLUNG RESEARCH

\author{
A. MAQUET, A. DUBOIS and V. VÉNIARD \\ Laboratoire de Chimie Physique (CNRS UA-176), Université Pierre \\ et Marie Curie, 11, Rue Pierre et Marie Curie, F-75231 Paris \\ Cedex 05, France
}

Résumé: Nous présentons les résultats de calculs théoriques nouveaux des amplitudes de transition et sections efficaces relatives à deux types distincts de processus associés au bremsstrahlung: a) Transitions stimulées à un photon (transitions "libre-libre") ayant lieu au cours de collisions électron-atome d' hydrogène, en présence d' un champ extérieur intense. b) Bremsstrahlung à deux photons, correspondant à l' émission spontanée de deux photons distincts lors d' une seule collision électron-atome. Les caractéristiques essentielles de ces processus sont mises en évidence et discutées.

Abstract: We report the results of new theoretical calculations of the transition amplitudes and cross-sections for two distinct bremsstrahlung-related processes: i) Stimulated one-photon transitions (Free-Free Transitions) occurring in the course of electron-H atom collisions, in the presence of a strong external field. ii) Two-photon Bremsstrahlung, corresponding to the spontaneous emission of two distinct photons in a single electron-atom collision event. The main features of these processes will be outlined and discussed.

\title{
1. Introduction.
}

The advent of powerful (soft) X-Ray sources $[1,2]$ and the development of coincidence techniques make possible to perform a new generation of Bremsstrahlung-related experiments. We will address here two distinct topics which open new perspectives onto the study of the dynamics of such electron-atom collisions, involving the exchange of one or several phatons with an external electromagnetic field. More precisely we will report recent advances we have contributed to in the theoretical description of the following radiative collision processes:

i) The so-called stimulated and inverse bremsstrahlung, which are expected to be observed in the course of the collision of electrons with an atomic target, in the presence of a 
strong external source. We will illustrate the main features of these stimulated processes with the help of a detailed calculation of the corresponding transition amplitudes and cross-sections in the simpler case of $\mathrm{e}^{-}-\mathrm{H}$ atom collisions. We will also evidence the differences existing between stimulated and conventional (spontaneous) bremsstrahlung.

ii) The two-photon Bremsstrahlung, corresponding to the simultaneous (spontaneous) emission of two distinct photons, occurring during the course of a single electron-atom collision event. Such a process, which has been recently observed in the X-ray range [3], can be interpreted as a second-order radiative transition taking place into the Coulomb continuum of the atomic nucleus. We will present here the results of an exact calculation of the corresponding non-relativistic dipole transition probabilities [4,5]. It is interesting to note that, although our results have been independently confirmed [6], notable discrepancies still persist between theoretical and experimental data.

In Section 2, we will briefly outline the theoretical framework and the formalism used in the description of the stimulated Free-Free Transitions (FFT's) occurring in the course of the collision of fast electrons with hydrogen atoms. The main results of our calculation, which relies on the use of compact representations of the Coulomb Green's function, will be presented in the Section 3, particular emphasis being given to the role of the atomic dynamic polarizability. In order to make clear the specificity of stimulated FFT's with respect to conventional (spontaneous) Bremsstrahlung, we will compare the relevant cross sections in Section 4. Such a comparison shows that the study of these stimulated transitions can provide more physical insight into the dynamics of such radiative processes than the one of spontaneous transitions. Our paper will end with a presentation and a short discussion of the main features of two-photon Bremsstrahlung. The possible origin of the above mentioned discrepancy between theory and experiment will be briefly addressed.

\section{Formalism}

Electrons colliding with atomic targets, in the presence of a strong external electromagnetic field, can pick up or lose one quantum of energy from the field. Such FFT's were primarily of astrophysical interest, until it has been recognized that they play also an important role in the absorption and stimulated emission of radiations from hot gases and plasmas [7]. Note that in the presence of very intense laser sources, multiphoton FFT's may become observable, which has given rise to an important specialized literature [8]. As we will show next, it is likely that, in the X-ray range, only the lowest-order (one-photon) transitions could be easily observed: we will accordingly restrict here the discussion to one-photon exchanges and model these processes with the help of the simpler $\mathrm{e}^{-}-\mathrm{H}$ atom system. Although this model may be thought to be somewhat naive, it displays in fact all the main features of the dynamics of such radiative collisions and permits in particular to discuss the relative importance of the atomic polarizability. 
This class of processes can be symbolically described by the equation:

$$
\mathrm{e}^{-}\left(\mathrm{E}_{\mathbf{j}}, \mathbf{k}_{\mathbf{i}}\right)+\mathrm{H}(1 \mathrm{~s}) \pm \gamma(\varepsilon, \omega) \rightarrow \mathrm{e}^{-}\left(\mathrm{E}_{\mathrm{f}}, \mathbf{k}_{\mathbf{f}}\right)+\mathrm{H}(1 \mathrm{~s}),
$$

where $\mathrm{E}_{\mathbf{i}}\left(\mathrm{E}_{\mathbf{f}}\right)$ and $\mathbf{k}_{\mathbf{i}}\left(\mathbf{k}_{\mathbf{f}}\right)$ are the energies and wave vectors of the incoming (outgoing) projectile; $H(1 s)$ represents the hydrogen atom in its ground state and $\pm \gamma(\varepsilon, \omega)$ is associated to the absorption $(+)$ or the stimulated emission (-) of one photon from the external field, with polarization $\varepsilon$ and frequency $\omega . E_{i}$ and $E_{f}$ are connected via the energy conservation relation (atomic units will be used throughout):

$$
\mathrm{E}_{\mathrm{i}} \pm \omega \quad=\mathrm{E}_{\mathrm{f}}
$$

The theoretical treatment of these processes presents considerable difficulties [9]. It does, however, simplify notably if one restricts oneself to the case of collisions involving fast (non-relativistic) electrons and assumes the validity of the electric dipole approximation as regards to the radiative step. The electron wave functions can thus be approximated by plane waves $\left(1^{\text {st }}\right.$ Born approximation) and exchange effects can be neglected. Within this framework, a simple perturbative analysis shows that the process is second order in the interaction hamiltonians [10]:

$$
H_{e}=\left(\frac{1}{r_{12}}-\frac{1}{r_{1}}\right) ; H_{r}=A_{0}\left(a+a^{\dagger}\right) \varepsilon \cdot p ;
$$

here $\mathrm{H}_{e}$ corresponds to the Coulomb interaction between the projectile and the atom, $\mathbf{r}_{1}$ being the position of the projectile and $r_{12}=\left|r_{1}-r_{2}\right|$, where $r_{2}$ is the position of the atomic electron; $H_{r}$ is the dipole interaction hamiltonian in which $A_{0}$ is the amplitude of the vector potential of the external field, with polarization $\varepsilon$ and $\mathrm{a}^{\dagger}$ and a are the usual photon creation and annihilation operators. The advantages of such a simplified perturbative treatment is that it allows to perform exact calculations and, accordingly, to discuss in a well defined test case, the physics of the process.

After some straightforward algebra, summing in particular over the intermediate free wave projectile states, one is left with an expression of the differential cross section which is expressed in terms of atomic matrix elements [11]:

$$
\begin{aligned}
& \frac{\mathrm{d} \sigma}{\mathrm{d} \Omega\left(\mathrm{k}_{\mathrm{f}}\right)}=\frac{8 \pi \alpha}{\Delta^{4}} \frac{\mathrm{k}_{\mathrm{f}}}{\omega}\left|\frac{\Delta . \varepsilon}{\omega}<1 \mathrm{~s}\right|\left(\mathrm{e}^{\mathrm{i} \Delta . \mathbf{r}}-1\right)|1 \mathrm{~s}\rangle+
\end{aligned}
$$

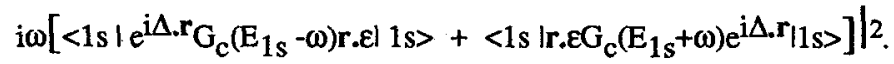

Here $\alpha$ is the fine structure constant, $\Delta=\mathbf{k}_{\mathbf{i}}-\mathbf{k}_{\mathbf{f}}$ is the momentum transfer and $\mathrm{G}_{\mathrm{c}}(\Omega)$ is the Coulomb Green's function and the cross section, defined after Geltman's [9], is proportional to a ${ }_{0}^{5}$, 
where $a_{0}$ is the Bohr radius. We note that each term in this expression can be associated to one of the Feynman diagrams displayed in the Fig. 1. More precisely the first term, in Eq.(4), corresponds to the diagrams numbered I and II which depict the photon emission (or absorption) as resulting from the interaction of the field with the electron projectile. Accordingly, this contribution will be referred to as "electronic" in the following. We note, for the sake of future discussion, that the term -1 , which appears here, represents the electron-nucleus interaction contribution to the process and will be named as "Coulomb". Finally, the last two terms, containing the Coulomb Green's function, correspond to the diagrams III and IV which are associated to the interaction of the atom with the radiation field, resulting in the exchange of one photon. Their corresponding contributions will be referred to as "atomic" in the following.

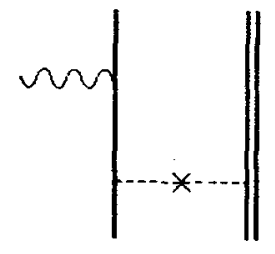

I

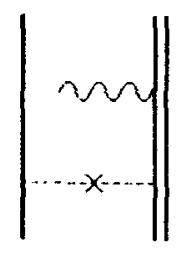

III

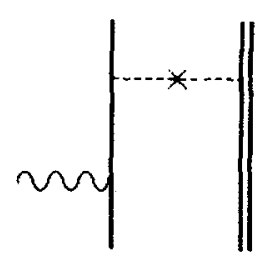

II

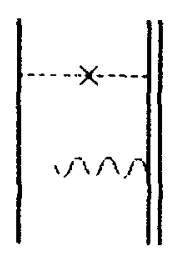

IV

Figure 1. Lowest-order Feynman diagrams contributing to one-photon exchange (FFT), occurring in the course of an electron- $\mathrm{H}$ atom collision. 
The calculation of these latter contributions represents obviously the main difficulty of the problem, since the other term reduces in fact to a trivial (ground state) atomic form factor. We have performed an exact analytical calculation of these second order matrix elements, by using Hostler's integral representation of the Coulomb Green's function [12]. Note that, as an independent check of our calculation, we have also used another numerical code, based on a sturmian expansion of the Coulomb Green's function [13]. Our main results are discussed in the next Section.

\section{Analysis of FFT's cross sections}

The evaluation of the exact analytical expressions so obtained permitted us to discuss in some detail the physics of the process (see, for instance, the reference [12]). Our findings may be summarized as follows:

In the relatively high kinetic energy range considered here for the incoming electron, the main parameters governing the dynamics of the problem are respectively the radiation frequency and the orientation of the polarization vector, with respect to the incoming and outgoing electron momenta. This relative orientation has a purely geometrical effect which, in the case considered here, is entirely contained in the overall factor $\Delta . \varepsilon=\left(\mathbf{k}_{\mathbf{i}}-\mathbf{k}_{\mathbf{f}}\right) . \varepsilon$ which naturally appears into the expression of the transition amplitude. The presence of this overall factor has important consequences on the variations of the differential cross sections: one notes in particular that this latter becomes identically zero at frequencies and scattering geometries such that $\Delta \perp \varepsilon$ (minimum denoted (a) in Fig. 2). We should emphasize, at this point, that such kinematical minima do not appear in the corresponding conventional (spontaneous) Bremsstrahlung differential cross sections, since these latter are obtained as resulting of an averaging procedure over the polarization states of the emitted light, procedure which washes out the minima.

Depending on the scattering geometry and photon frequency, another kind of minima may appear in the angular distribution or in the dispersion curves (see the minima denoted (b) in the Fig. 2). Their origin can be ascribed to a destructive interference phenomenon between the "atomic" and "electronic" contributions, into the overall amplitude [12]. These minima do exist also in the angular distributions (not shown here), in a wide range of scattering geometries and photon frequencies and their presence (absence) provide a nice indication on the relative magnitudes of the "atomic" and "electronic" terms in the expression Eq. (4). Interestingly enough, it seems that such minima are not an artefact specific to our simplified approach and would survive even if more refined models, including for instance higher order Born corrections, were to be used [14]. It should be noted however that, again, these features are washed out in the corresponding conventional Bremsstrahlung spectra, in which the propagation direction of the outgoing electron is not recorded.

The dispersion curve for the differential cross section presented in the Fig. 2, 


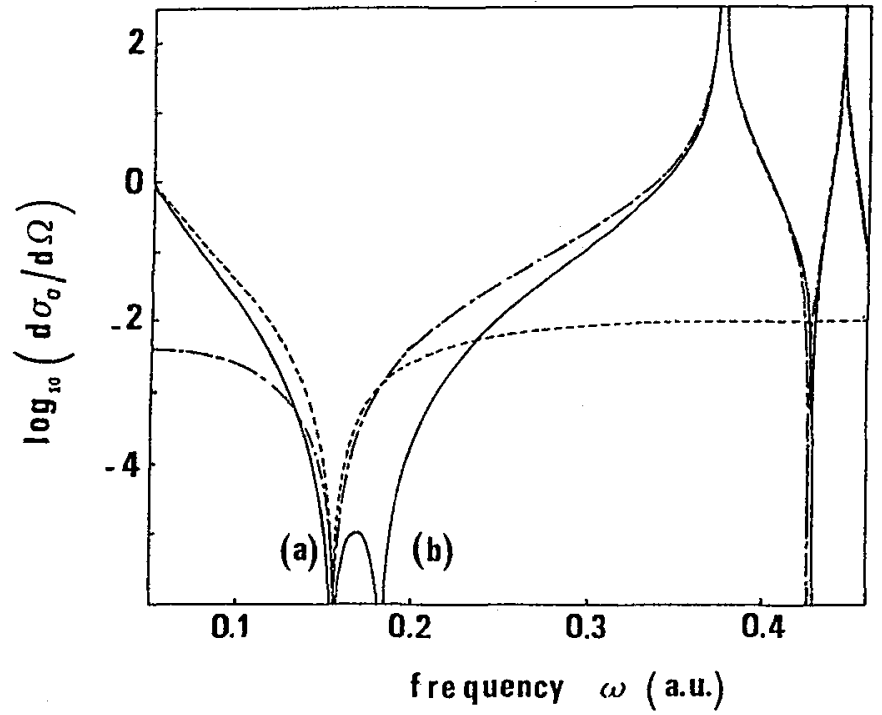

Fig. 2. Typical variations of the FFT differential cross section in. terms of the external field frequency. $\vec{\varepsilon} / / \vec{k}_{f},\left(\vec{k}_{i}, \vec{k}_{f}\right)=10^{\circ}, E_{i}=5 . a u$. Dashed line: contribution of the "electronic" teirms ; dot-dashed line: contribution of the "atomic" terms ; solid line : total cross section.

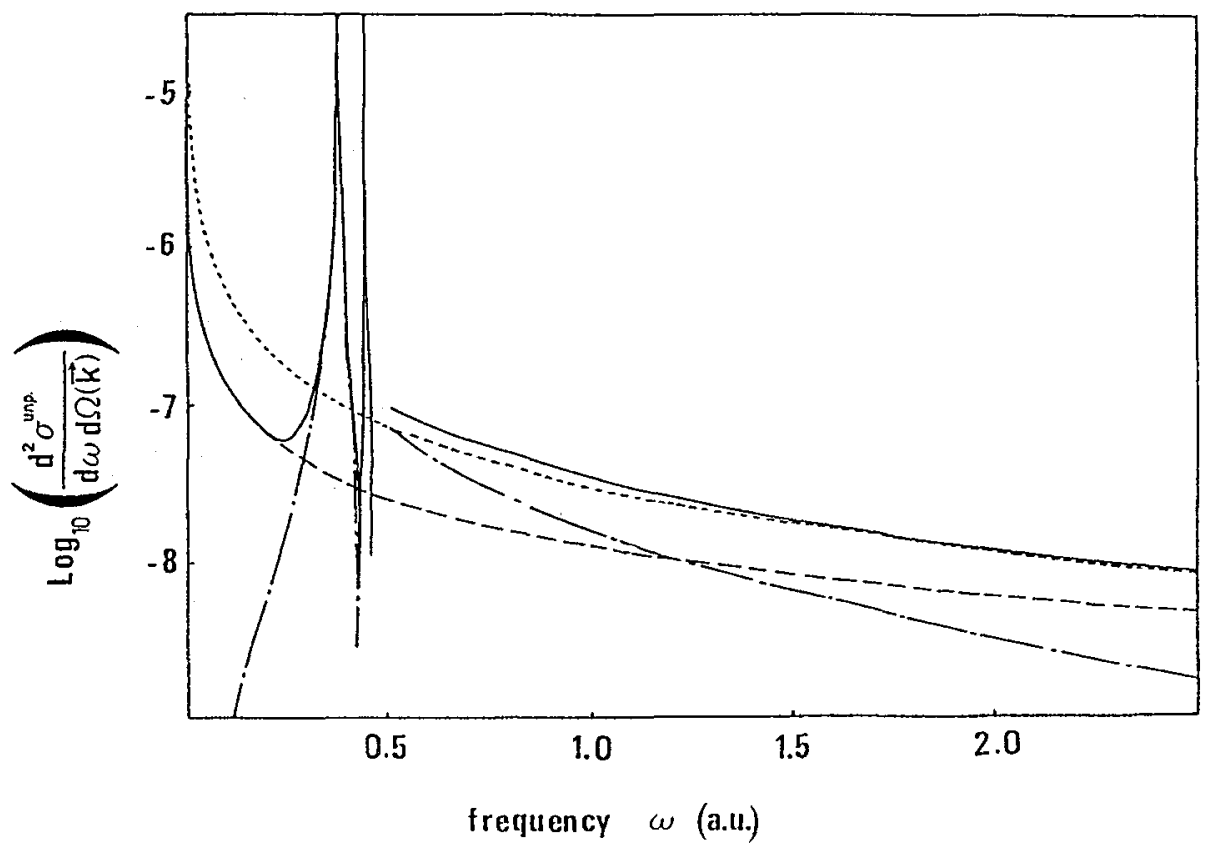

Fig. 3. Typical bremsstrahlung differential cross section for the detection of an unpolarized photon $\omega$, within $d \Omega(k), E_{i}=10$ au. $\theta_{k}=45^{\circ}$ Dotted line : "Coulomb" contribution; dashed line "electronic" "contribution; dot-dashed line : "atomic" contribution ; solid line : total cross section. 
displays also some interesting general features of FFT's. One observes in particular that, still at the relatively high projectile energies considered here, the "electronic" contribution (diagrams I and II, Fig. 1) is dominant in the low frequency domain. This is in qualitative agreement with the so-called Low theorem, connected to the infrared divergence of the Bremsstrahlung cross section and which precisely states that, in the soft-photon limit, the cross section diverges and becomes also proportional to the elastic scattering one [15].

In addition to the already discussed destructive interferences, the "atomic" terms play an important role, as expected, in the range of frequencies close to characteristic atomic excitation frequencies. On the contrary, at higher photon energies, the "Coulomb" contribution, associated to the electron-nucleus interaction and contained into the "electronic" term, provides the dominant contribution to the FFT spectrum. This is consistent with the fact that screening effects become less important as the photon frequency is higher. Similar general conclusions may be drawn from the study of the Bremsstrahlung cross sections, as we will show below.

\section{FFT's vs. Bremsstrahlung.}

As we have already mentioned in the preceding Section, FFT cross sections contain more structures (thus more informations) than the corresponding conventional Bremsstrahlung ones. This can be readily verified in the Fig. 3 in which we have reported the doubly differential Bremsstrahlung cross section for the emission of one photon into the solid angle $d \Omega(k)$ with an energy such that: $\omega=k_{i}^{2} / 2-k_{f}^{2} / 2$. By comparing with the Fig. 2 , one observes that the kinematic and interference minima are not present anymore, as a result of the integration over the final electron direction and the averaging over the polarization states of the emitted light. As already noted by several authors $[11,16]$, the only features to survive are then the resonances corresponding to the excitation frequencies of the atom.

It is important to note that the cross sections for FFT's (Fig. 2) and for (spontaneous) Bremsstrahlung (Fig. 3) are not defined in the same way and, in particular, have not the same dimensions [17]. Accordingly their magnitudes should not be compared without precautions. There are however significant physical differences between those cross sections, which are well exemplified by the elementary expressions one can derive in the pure Coulomb case, in the first Born approximation, for the emission of one photon of frequency $\omega$ (in whatever direction) and the scattering of the projectile into the solid angle $d \Omega\left(k_{\mathbf{f}}\right)$ :

i) FFT (the photons are not detected)

$$
\frac{d \sigma_{B}}{d \Omega\left(k_{f}\right)}=\frac{16 I}{\omega^{4}} \frac{k_{f}}{k_{i}} \frac{(\Delta \varepsilon)^{2}}{\Delta^{4}} Z^{2}
$$


Here $\mathrm{Z}$ is the charge of the nucleus, the index $\mathrm{B}$ stands for the first Born approximation and $\mathrm{I}$ is the intensity of the external source expressed in atomic units, the (time averaged) atomic unit of intensity being $\mathrm{I}_{0}=3.510^{16} \mathrm{~W} / \mathrm{cm}^{2}$.

ii) Bremsstrahlung (for the sake of comparison with the preceding formula, in addition to sum over the polarization states, we have integrated over the propagation direction of the emitted photon); the doubly differential cross section reads:

$$
\frac{d \sigma_{B}}{d \Omega\left(k_{f}\right) d \omega}=\frac{8}{3 \pi} \frac{\alpha^{3}}{\omega} \frac{k_{f}}{k_{i}} \frac{Z^{2}}{\Delta^{2}}
$$

We note first the important difference between the angular distributions for the scattered electron: in the conventional Bremsstrahlung case it is isotropic, Eq. (6), whereas it is proportional to $(\Delta . \varepsilon)^{2}$, i.e. is strongly dependent on the scattering geometry, in the stimulated case, Eq. (5). We observe also that, although both cross sections are divergent in the soft-photon limit [15], the $\omega^{-4}$ dependence of the cross sections for stimulated processes makes these latter much more probable in the low frequency regime than in the $\mathrm{X}$-ray range. This tendency is even more accentuated when considering higher-order (multiphoton) transitions $[4,8,17]$, which means that it is likely that only one-photon FFT's will be easily observed in the $\mathrm{X}$-ray domain, unless very high intensities could be reached. As a final comment, we would like to stress that the main conclusions of this simple analysis, based on a first Born calculation, are still verified, at least in a broad sense, even when considering more realistic descriptions of the process.

\section{Two-photon Bremsstrahlung.}

Such a process, corresponding to the spontaneous emission of two distinct photons in a single electron-nucleus collision event, was predicted to arise as a radiative correction to one-photon bremsstrahlung, since the early days of the Quantum Electrodynamics theory. It can be conveniently represented as follows:

$$
\mathrm{e}^{-}\left(\mathbf{k}_{\mathbf{i}}\right)+(\text { nucleus }) \rightarrow \mathrm{e}^{-}\left(\mathbf{k}_{\mathbf{f}}\right)+(\text { nucleus })+\gamma\left(\omega_{1}\right)+\gamma\left(\omega_{2}\right)
$$

with the conservation energy: $k_{i}^{2} / 2=k_{f}^{2} / 2+\omega_{1}+\omega_{2}$

However, as the corresponding probabilities are easily estimated to be much smaller, i.e. about $\alpha=1 / 137$ times, than in the one-photon case, the two-photon spectrum was expected to be completely buried into a dominant bremsstrahlung emission background. Accordingly, the theoretical study of the process was, for a long time, considered as a somewhat academic problem 


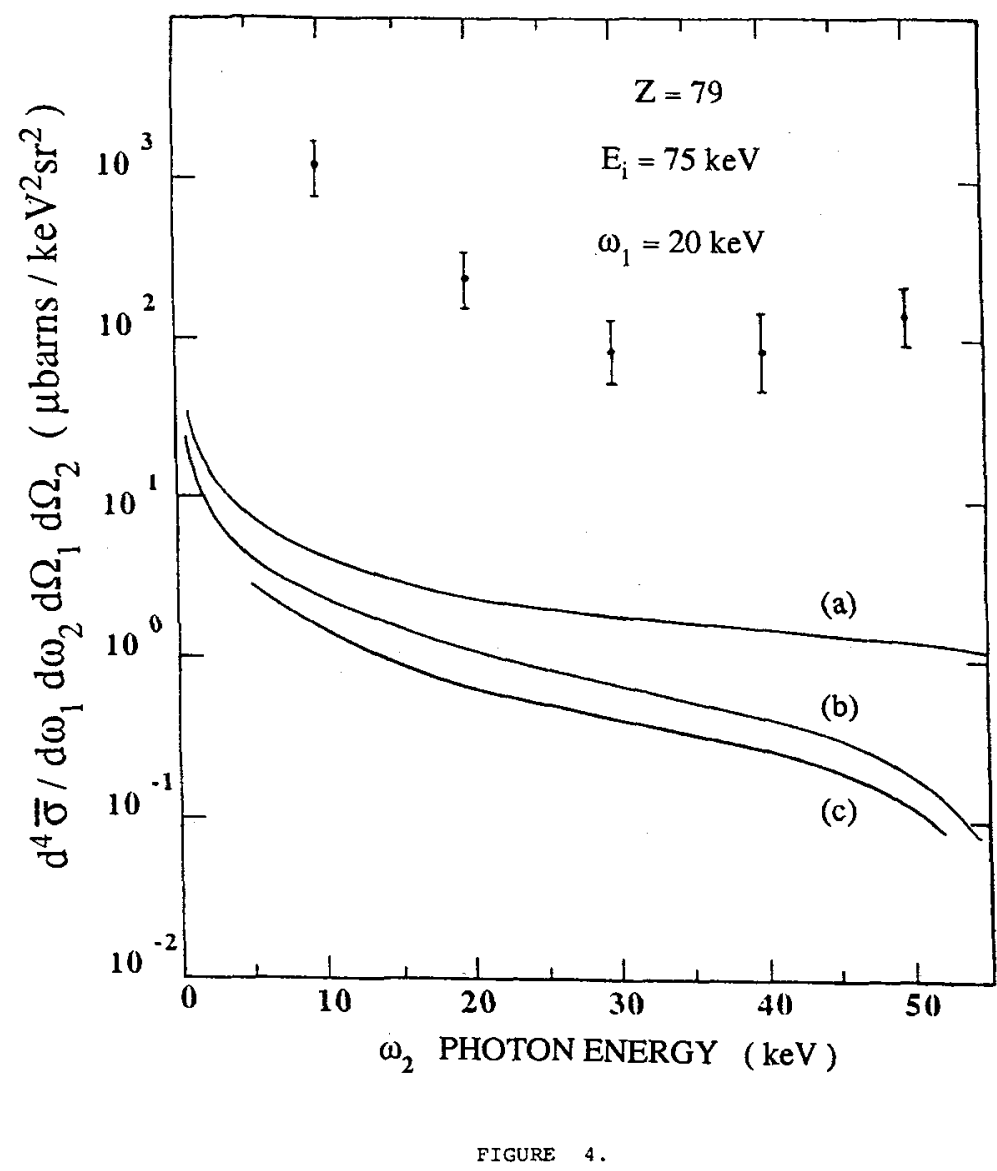

Fig. 4. Comparison of the experimental results ref. (3) with theory for two-photon bremsstrahlung cross sections. (a) our results ; (b) nonrelativistic 1st Born approximation ; (c) relativictic 1st Born approximation.

until recent experimental efforts $[3,20]$, stimulated a renewal of interest for the theory of the subject [4-6].

Standard time-dependent perturbation theory leads straightforwardly to express the relevant transition probability in terms of a second-order atomic matrix element between two Coulomb continuum states. Within the framework of the nonrelativistic electric dipole approximation, we were able to compute exactly these matrix elements, for the first time $[4,5,18]$. Our calculation rely on the use of Schwinger's integral representation of the Coulomb Green's function [19], and we have pushed the analytic calculation as far as possible. Our numerical results, which have been confirmed independently by Florescu et al. [6], disagree notably with the preliminary experimental data reported so far, see Fig. (4). A detailed theoretical analysis shows that it is likely that even more sophisticated models, taking consistently into account relativistic as well as atomic structure effects (screening, for instance), would not fill the gap. It is thus interesting to note that more refined experiments are currently under way and that the agreement is already improved [20]. 


\section{Conclusions.}

The salient features of this short presentation of FFT's and two-photon bremsstrahlung can be summarized as follows:

-Although higher frequencies are not favored, one-photon FFT's will probably be observed in the presence of strong X-ray sources, specially in the soft X-ray domain.

-The corresponding cross sections are much sensitive to the scattering geometry and to the atomic polarisability. Accordingly, they contain more informations than the corresponding conventional (spontaneous) bremsstrahlung spectra.

-The analytic calculation and the numerical computation of second-order dipole transition matrix elements between Coulomb continuum states are now well mastered.

\section{Acknowledgements.}

Parts of the results presented here are the outcome of fruitful collaborations with Pr. J. Broad, Pr. M. Gavrila and Dr. S. Jetzke to whom the authors express their grateful thanks. The autors are also indebted to Pr. F. H. M. Faisal for helpful discussions and who also kindly brought to their attention the earlier reference to the work by Maria Göppert Mayer [10].

\section{References.}

[1] P. Jaegle, 14 $4^{\text {th }}$ International Conference on X-ray and Inner-shell Processes, X-87 (Paris, 1987); this Volume.

[2] R. Haensel, $14^{\text {th }}$ International Conference on X-ray and Inner-shell Processes, X-87 (Paris, 1987); this Volume.

[3] J. C. Altman and C. A. Quarles, Phys. Rev. A 31, 2744 (1985); ibid. Nucl. Inst. Meth. A 240, 538 (1985).

[4] V. Véniard, Thesis (Université Pierre et Marie Curie,Paris, 1986), unpublished.

[5] V. Véniard, M. Gavrila and A. Maquet, Phys. Rev. A 35, 448 (1987).

[6] V. Florescu and V. Djamo, Phys. Lett. A119, 79 (1986), see also , $14^{\text {th }}$ International Conference on X-ray and Inner-shell Processes, X-87 (Paris, 1987); Abstracts of Contributed Papers Ab 37.

[7] M. Gavrila and M. van der Wiel, Comments At. Mol. Phys. 8, 1 (1978).

[8] Recent reviews on multiphoton FFT's include: L. Rosenberg, in Advances in Atomic and Molecular Physics edited by D. R. Bates and B. Bederson (Academic, New York, 1982) Vol 18,p1;A. Weingartshofer and C. Jung, in Multiphoton Ionization of Atoms, edited by S. L. Chin and P. Lambropoulos (Academic, New York, 1984) p155.

[9] See, for instance: M. J. Conneely and S. Geltman, J. Phys. B 14, 4847 (1981) and references therein.

[10] This approach had been explicitly formulated in the pioneeering work by M. Göppert Mayer, Ann. Phys. (Leipzig), 9, 273 (1931). It has been rederived by V. M. Buimistrov, Phys. Lett. A30, 136 (1969). 
[11] V. M. Buimistrov and L. I. Trakhtenberg, Sov. Phys. JETP, 42, 54 (1975); N. K. Rahman and F. H. M. Faisal, J. Phys. B 9, L275 (1976); ibid. 11, 2003 (1978); A. Lami and N. K. Rahman, ibid. 16, L201 (1983).

[12] A. Dubois, A. Maquet and S. Jetzke, Phys. Rev. A34, 1888 (1986).

[13] S. Jetzke, J. Broad and A. Maquet, J. Phys. B 20, 2887 (1987).

[14] C. Joachain, private communication.

[15] F. E. Low, Phys. Rev. 110, 974 (1958).

[16] M. Ya. Amusia, A. S. Baltenkov and A. A. Paiziev, JETP Lett. 24, 332 (1976);G. Wendin and K. Nuroh, Phys. Rev. Lett. 39, 48 (1977); for a recent review, see for instance: M. Ya. Amusia, Comments At. Mol. Phys. 11, 123 (1982). Relativistic effects are discussed in: M. Ya. Amusia, M. Yu. Kuchiev, A. V. Korol' and A. V. Solov'ev, Sov Phys. JETP 61, 224 (1985).

[17] The FFT cross sections are often defined as having the dimension of (length) ${ }^{5}$ in astrophysics and sometimes also as usual scattering cross section with the dimension (length) ${ }^{2}$. By contrast, bremsstrahlung cross sections are defined within an unit interval of energy and accordingly have often the dimension of (length) ${ }^{2}$ /energy.

[18] M. Gavrila, A. Maquet and V. Véniard, Phys. Rev. A 32, 2537 (1985).

[19] J. Schwinger, J. Math. Phys. 5, 1606 (1964).

[20] C. A.Quarles and H. E. Lehtihet, in Abstracts of Contributed Papers, $15^{\text {th }}$ International Conference on the Physics of Electronic and Atomic Collisions (Brighton UK, 1987) p.190. 